\title{
Is arthritis associated with cardiovascular diseases in both sexes? An analysis of NHANES data
}

\author{
Rachelle Saade ${ }^{1}$, Danielle Laurin ${ }^{2,3}$ and Clermont E Dionne ${ }^{1,2,4 *}$ \\ ${ }^{1}$ Department of Social and Preventive Medicine, Faculty of Medicine, Laval University, Quebec City, QC, G1V 0A6, Canada \\ ${ }^{2}$ Centre d'excellence sur le vieillissement de Québec, Centre de recherche du CHU de Québec-Université Laval, Quebec City, QC, G1S 4L8, Canada \\ ${ }^{3}$ Faculty of Pharmacy, Laval University, Quebec City, QC, G1V 0A6, Canada \\ ${ }^{4}$ Department of Rehabilitation, Faculty of Medicine, Laval University, Quebec City, QC, G1V 0A6, Canada
}

\begin{abstract}
Background: Among arthritis patients, cardiovascular morbidity is increased, and sex disparities exist in cardiovascular diseases and arthritis. Thus, the hypothesis that the association between cardiovascular diseases and arthritis might vary according to sex seems plausible.

Aim: To examine the relationship of arthritis with the most frequently diagnosed cardiovascular diseases, and to check if sex modifies this association.

Methods: We analyzed data from a large representative sample of the U.S. population: The National Health and Nutrition Examination Survey (NHANES) 20052006. Our cross-sectional analyses included 3621 participants aged between 20 and 69 years old. Self-reported diagnoses of arthritis (main independent variable) and cardiovascular diseases (dependent variable) were measured during face-to-face interviews, and different covariates were identified as potential confounders for the association examined. Analyses were stratified by sex.

Results: In multivariate analyses, a significant positive association was found between arthritis and cardiovascular diseases (OR: 2.01; 95\% CI: 1.38-2.94; $\mathrm{p}$ value $=0.0003$ ), but there was no effect modification by sex (men: OR: 1.95; 95\% CI: 1.32-2.87/ women: OR: 2.38; 95\% CI: 0.96-5.86) (p=0,729).

Conclusions: In this large cross-sectional study, we observed a statistically significant association between self-reported diagnoses of arthritis and cardiovascular diseases in a cohort of non-institutionalized U.S. adults. The association persisted after adjustment for several confounders but was not modified by sex.
\end{abstract}

Abbreviations: NHANES: National Health and Nutrition Examination Survey; CVD: Cardiovascular Diseases; BMI: Body Mass Index; SD: Standard Deviation; OR: Odds Ratio; CRP: C - Reactive Protein; MEC: Mobile Examination Centers.

\section{Introduction}

Cardiovascular diseases (CVD) are responsible for increasing morbidity and mortality worldwide [1,2]. They are bringing about a heavy burden on the economy, especially in low and middle-income countries [3]. Prevention of CVD relies on lowering modifiable risk factors such as hypertension, diabetes, hyperlipidemia and adverse lifestyle factors [1] (alcohol consumption, cigarette smoking, physical inactivity, etc.) [1,4]. Some biomarkers related to hemostasis and inflammation, such as C-reactive protein (CRP), have a strong predictive value for CVD [2,5-8].

Arthritis includes several rheumatic diseases and disorders that affect the joints and the surrounding connective tissues [9]. Inflammation, pain, redness and stiffness in and around one or more joints are considered the cardinal symptoms of arthritis [10,11]. According to the 2003 U.S. National Health Interview Survey, 25\% of adults will have a diagnosis of arthritis by the year 2030, with an estimated $37 \%$ of them reporting arthritis-attributable activity limitations.

Among arthritis patients, disease activity and severity are associated to cardiovascular risk through inflammatory biomarkers, especially CRP [12,13]. Interleukin-6 (IL-6) is produced by inflammatory cells [6], and regulates the expression of CRP [5,7]. Evidence suggests that CRP is involved in the initiation and progression of atherosclerotic lesions, contributing to the development of cardiac ischemic syndromes [14]. Cardiovascular morbidity is thus increased in rheumatoid arthritis patients [13], and cardiovascular risk factors such as smoking, hypertension or obesity are overrepresented among patients with chronic rheumatic diseases when compared to the general population [4].

Inflammatory biomarkers could modulate the association between arthritis and CVD, but their physiological relevance remains not totally understood [15]. It is also possible that arthritis constitutes an independent risk factor for CVD by itself. It is thus important to investigate the association between arthritis and CVD to help reduce the CVD-related morbidity and mortality by improving control measures for arthritis.

There exist important sex disparities in CVD [16-19] and arthritis $[20,21]$. Men develop CVD 7 to 10 years before women in average

*Correspondence to: Clermont E. Dionne, Centre d'excellence sur le vieillissement de Québec, Centre de recherche du CHU de Québec-Université Laval, Quebec City, QC, G1S 4L8, Canada, E-mail: clermont.dionne@ crchudequebec.ulaval.ca

Key words: arthritis, cardiovascular diseases, sex effect, NHANES 2005-2006

Received: January 12, 2021; Accepted: Janauary 22, 2021; Published: Janauary 30,2021 
$[16,17]$. Sex differences are important in the metabolic syndrome and inflammatory reaction and have a specific role in the manifestation of CVD [16]. Generally, women have worse inflammatory disease activity [21], report more severe symptoms and have higher work disability percentages, while men have higher mortality rates than women [20]. Also, arthritis is more prevalent among women than among men [10]; $60 \%$ of people with arthritis are women and most types of arthritis are more common in women, including osteoarthritis, rheumatoid arthritis, and fibromyalgia; however, gout is more common in men [22]. Experts don't know exactly why women are at higher risk of developing most types of arthritis, or why men are at higher risk of developing gout. Though CVD risk factors are shared by men and women, some may be more prevalent and/or more significant for one sex; for example, having diabetes may be a stronger risk for certain types of CVD in women. Also, women tend to develop CVD at an older age than men $[23,24]$. It thus seems plausible that the association between CVD and arthritis varies according to sex (effect modification by sex).

To our knowledge, no study has yet tested this hypothesis. Shedding light on a possible effect modification of the association between arthritis and CVD by sex would provide better understanding of the frequency and risk of CVD among persons with arthritis and would thus help healthcare professionals better adapt CVD prevention and treatment to individual characteristics.

The objectives of this study were to examine the relationship of arthritis with the most frequently diagnosed CVD, and to verify if sex modifies this association.

\section{Methods}

\section{Source of data}

Data came from a large representative sample of the U.S. population: The National Health and Nutrition Examination Survey (NHANES) 2005-2006. The National Center for Health Statistics of the Centers for Disease Control and Prevention of the United States [25] is conducting the NHANES surveys each year. They consist in national cross-sectional health surveys designed to obtain information on the health and nutritional status of the non-institutionalized U.S. population. Aiming to provide estimates that could be generalized to the entire U.S. population, NHANES uses a complex, stratified, multistage probability cluster design. Every participant is considered to represent 50,000 U.S. residents. All participants provided written informed consent, and data collection was based on standardized health questionnaires administered during respondents' home interview, with detailed physical examination and blood collection performed in wellequipped mobile examination centers (MEC), that travelled to different sites all across the country, or at the participant's home [25].

\section{Sample selection}

The inclusion criteria for this study were to have participated to NHANES 2005-2006 and to be aged between 20 and 69 years. Exclusion criteria were mainly linked to the blood samples: 1) having hemophilia, 2) having received chemotherapy in the last 4 weeks, 3) presence of the following on two arms: rashes, gauze dressings, casts, edema, paralysis, open wounds, missing limbs, sclerotic veins, allergies to cleansing agents, burns or scar tissues. Pregnant women were also excluded to eliminate hypertension or other CVD induced by pregnancy [25].

Figure 1 shows the detail of participant selection for this study. Overall, 10,348 individuals participated to NHANES 2005-2006. Of these, 382 were pregnant women, 5,323 were younger than 20 years, 882 were aged 70 years or more, and 140 did not complete the physical

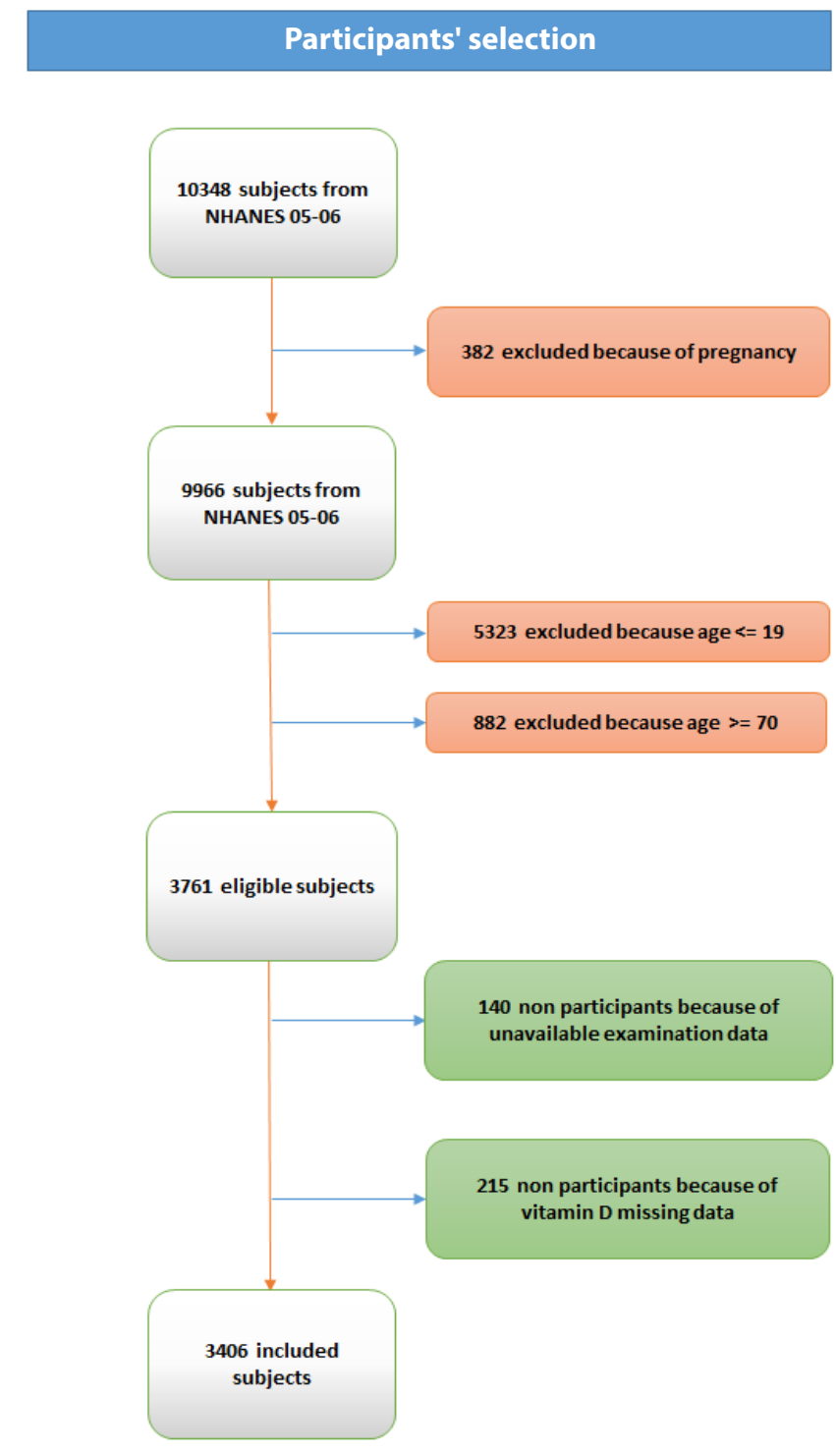

Figure 1. Participants' selection schematization

examination. The final sample size of the current study was 3,621 (96.3\% of those eligible).

Main independent and dependent variables: The NHANES 2005-2006 survey included a "Medical Conditions Questionnaire" in which participants were asked to answer questions about several health conditions. Our main independent variable was the presence of arthritis (yes/no) as reported in the question "Has a doctor or another health professional ever told you that you had arthritis?" Participants were also asked "Has a doctor or another health professional ever told you that you had congestive heart failure?"; "- coronary heart disease?"; “- angina pectoris?”; “- a heart attack?”; “- a stroke?” The dependent variable was defined as a history of CVD (yes/no) if they answered "Yes" to at least one of these questions. These measures have been used successfully before to estimate the prevalence of CVD [26] in the United States.

Other independent variables: Several variables were used to describe the participants' sociodemographic, lifestyle and physical 
characteristics. Some of these were identified as potential confounders: age, sex, ethnicity, body mass index (BMI), smoking status, alcohol consumption, kidney and liver diseases, cholesterol and triglyceride blood levels, diabetes mellitus and hypertension. The choice of these covariates was based on the literature [13,27-29].

Ethnicity was considered into five categories: Mexican American, other Hispanic, non-Hispanic white, non-Hispanic black, and other (including multi-racial). BMI was calculated as weight in kilograms divided by the square of height in meters and classified into 4 categories: underweight (BMI <18.5), normal (18.5 $\leq$ BMI <24.9), overweight $(24.9 \leq \mathrm{BMI}<30)$, and obese (BMI $\geq 30)$. Smoking status was reflected by serum concentrations of cotinine provided from the laboratory data file. Serum cotinine was measured by an isotope dilution-high performance liquid chromatography/atmospheric pressure chemical ionization tandem mass spectroscopy (ID HPLC-APCI MS/MS) [30]. For analyses, cotinine serum levels were classified to create the following smoking groups: non-smokers/non exposed $(0.011$ $\leq$ cotinine $\leq 1)$, non-smokers/exposed $(1<$ cotinine $\leq 15)$ and smokers/ exposed (cotinine $>15$ ) [31]. Alcohol consumption was calculated as the average number of weekly drinks self-reported by the participants. Light consumption was defined as less than 3 drinks per week for both men and women, moderate consumption as between 3 and 14 drinks per week for men and between 3 and 7 drinks per week for women, and vigorous consumption as more than 14 drinks per week for men and more than 7 drinks per week for women. Self-reported data on chronic diseases were available in the questionnaire data file. Diabetes mellitus, kidney and liver diseases were assessed by asking participants whether a doctor or another healthcare professional had ever told them they had these conditions. Serum total cholesterol, HDL-cholesterol, LDL-cholesterol and triglycerides were measured enzymatically using the Roche Hitachi 717 in 2005 and the Roche Hitachi 717 and 912 in 2006, but no adjustment was necessary for the instrumentation change $[32,33]$. They were considered as continuous variables in analyses. Hypertension assessment was based on blood pressure readings performed during the survey examination process. Up to four readings of systolic and diastolic blood pressure were obtained from participants in the MEC. We calculated the mean of the first 3 measurements of systolic and diastolic blood pressure to generate participant's blood pressure, and categorized them as follows: normal (systolic blood pressure $<120 \mathrm{~mm} \mathrm{Hg}$ and diastolic blood pressure $<80 \mathrm{~mm} \mathrm{Hg}$ ), pre-hypertension (systolic blood pressure $\geq 120 \mathrm{~mm} \mathrm{Hg}$ and $<140$ $\mathrm{mm} \mathrm{Hg}$ or diastolic blood pressure $\geq 80 \mathrm{~mm} \mathrm{Hg}$ and $<90 \mathrm{~mm} \mathrm{Hg}$ ), hypertension stage 1 (systolic blood pressure $\geq 140 \mathrm{~mm} \mathrm{Hg}$ and $<160$ $\mathrm{mm} \mathrm{Hg}$ or diastolic blood pressure $\geq 90 \mathrm{~mm} \mathrm{Hg}$ and $<100 \mathrm{~mm} \mathrm{Hg}$ ), and hypertension stage 2 (systolic blood pressure $\geq 160$ or diastolic blood pressure $\geq 100 \mathrm{~mm} \mathrm{Hg}$ ). Physical activity was self-reported and categorized into 3 groups: light activity (when, over the last 30 days, participant walked or bicycled as part of getting to and from work, or school, or to do errands), moderate activity (when over the last 30 days, participant practiced an activity for at least 10 minutes that causes only light sweating or a slight to moderate increase in breathing or heart rate) and vigorous activity (when, over the last 30 days, participant practiced an activity that causes heavy sweating or large increases in breathing or heart rate).

\section{Statistical analyses}

NHANES recommends weighting the sample data to get better representation of the civilian noninstitutionalized U.S. population by producing estimates of statistics that would have been obtained if the entire population was surveyed [34]. Weighting measures were applied for all the survey analysis procedures. Values are expressed as weighted mean \pm standard deviation (SD) for normally distributed variables. Baseline demographics and clinical characteristics were compared between arthritis and non-arthritis groups using chi-square weighted test. Bivariate logistic regression models included only the main independent variable (arthritis) and the dependent variable (CVD). In multivariate logistic regression, we then adjusted for confounders. Weighted odds ratios (OR) with 95\% confidence intervals were calculated. A $p$ value $<0.05$ was considered significant. A full regression model included all potential risk factors for CVD. The backward method was used to evaluate the potential confounding effect of age, sex, body mass index, cholesterol (total, HDL and LDL), triglycerides, CRP and medical conditions. A variable was considered a confounder if it brought about a change in the OR of $\geq 10 \%$ compared with that for the full model. The final model included age, BMI, alcohol consumption and total cholesterol concentration as confounding variables. Finally, to test the modifying effect of sex on the relationship between arthritis and CVD, an interaction term between arthritis and sex was created and introduced in the final model. The SAS software (version 9.3) was used for statistical analyses.

\section{Results}

\section{Descriptive analyses}

Among the 3,621 subjects of the current study, 757 (20.9\%) reported having been diagnosed with arthritis. Selected subjects' characteristics according to arthritis status are shown in table 1, with $\mathrm{p}$ values of unadjusted comparisons between both groups for each characteristic. Study participants had a mean age of 42.7 years $(\mathrm{SE}=0.4)$, they were $51.3 \%$ male and $48.7 \%$ female, and Non-Hispanics Whites were the most represented (45.2\%). The majority of participants were nonsmokers (65.3\%), had light alcohol consumption (74.6\%) and said they had practiced vigorous physical activity in the past 30 days (55.5\%). Kidney (2.4\%) and liver (2.3\%) problems were rare; diabetes was more frequent (9.2\%). A diagnosis of CVD was reported by $7.2 \%$. Compared to subjects without arthritis, subjects with arthritis were significantly older, more often non-hispanic White, female, and obese. They reported less vigorous physical activity but were also less inactive than those without arthritis. Subjects with arthritis showed higher proportions of clinically raised CRP $(\mathrm{CRP}>1 \mathrm{mg} / \mathrm{dL})$, total cholesterol and triglycerides concentrations, diabetes mellitus and stages 1 and 2 hypertensions, than those without arthritis. Among subjects with arthritis, $18.1 \%$ reported a diagnosis of CVD compared to $4.4 \%$ of those without arthritis.

\section{Association of arthritis with CVD}

Table 2 shows the bivariate and the final multivariate logistic regression models. In bivariate analyses, a significant positive association was found between arthritis and CVD (OR: 4.81; 95\% CI: 3.66-6.31).

The final multivariate regression model included age, sex, BMI, alcohol consumption and total cholesterol concentrations as confounding factors. The association remained positive and statistically significant after adjustment (OR: 2.01; 95\% CI: 1.38-2.94).

The association between arthritis and CVD was statistically significant in men. The odds of CVD in men with arthritis was 1.95 times the odds of CVD in those without diagnosed arthritis. The $95 \%$ confidence interval $(1.32-2.87)$ excluded the null value. The odds of CVD in women with arthritis was 2.38 times the odds of CVD in those 
Table 1. Selected subjects' characteristics*.

\begin{tabular}{|c|c|c|c|c|}
\hline & $\begin{array}{l}\text { Study sample } n=3621 \\
\text { [missing values] }\end{array}$ & $\begin{array}{c}\text { Without arthritis } n=2864 \\
\text { [missing values] }\end{array}$ & $\begin{array}{l}\text { With arthritis } n=757 \\
\text { [missing values] }\end{array}$ & $P$ value ** \\
\hline Age (years) & $42.7 \pm 0.4$ & $40.2 \pm 0.4$ & $52.24 \pm 0.6$ & $<.0001$ \\
\hline Ethnicity & $3621[0]$ & $2864[0]$ & $757[0]$ & $<.0001$ \\
\hline Mexico-Americans & $781(21.6)$ & $690(24.1)$ & $91(12)$ & \\
\hline Other Hispanics & $125(3.5)$ & $109(3.8)$ & $16(2.1)$ & \\
\hline Non-Hispanics whites & $1638(45.2)$ & $1228(42.9)$ & $410(54.2)$ & \\
\hline Non-Hispanics blacks & $917(25.3)$ & $711(24.8)$ & $206(27.2)$ & \\
\hline Other race (including multi-racial) & $160(4.4)$ & $126(4.3)$ & $34(4.5)$ & \\
\hline Sex & $3621[0]$ & $2864[0]$ & $757[0]$ & $<.0001$ \\
\hline Male & $1856(51.3)$ & $1538(53.7)$ & $318(42.0)$ & \\
\hline Female & $1765(48.7)$ & $1326(46.3)$ & $439(58.0)$ & \\
\hline Body Mass Index (BMI) & $3572[49]$ & $2826[38]$ & $746[11]$ & $<.0001$ \\
\hline Underweight $(\mathrm{BMI}<18.5)$ & $60(1.7)$ & $50(1.8)$ & $10(1.3)$ & \\
\hline Normal $(18.5<=\mathrm{BMI}<24.9)$ & $976(27.3)$ & $842(29.8)$ & $134(18.0)$ & \\
\hline Overweight $(24.9<=\mathrm{BMI}<30)$ & $1214(34.0)$ & $1007(35.6)$ & $207(27.8)$ & \\
\hline Obese $(\mathrm{BMI}>=30)$ & $1322(37.0)$ & $927(32.8)$ & $395(52.9)$ & \\
\hline Smoking & $3399[222]$ & $2677[187]$ & $722[35]$ & 0.4951 \\
\hline Non-smokers/non-exposed $(0.011<=$ cotinine $<=1)$ & $2221(65.3)$ & $1758(65.7)$ & $463(64.1)$ & \\
\hline Non-smokers/exposed $(1<$ cotinine $<=15)$ & $184(5.4)$ & $149(5.6)$ & $35(4.9)$ & \\
\hline Smokers/exposed (cotinine> $>15$ ) & $994(29.3)$ & $770(28.7)$ & $224(31.0)$ & \\
\hline Alcohol consumption & $3300[321]$ & $2587[277]$ & $713[44]$ & 0.0042 \\
\hline Light consumption & $2460(74.6)$ & $1894(73.2)$ & $566(79.4)$ & \\
\hline Moderate consumption & $516(15.6)$ & $421(16.3)$ & $95(13.3)$ & \\
\hline Vigorous consumption & $324(9.8)$ & $272(10.5)$ & $52(7.3)$ & \\
\hline Physical activity & $3621[0]$ & $2864[0]$ & $757[0]$ & $<.0001$ \\
\hline Inactive & $1058(29.2)$ & $774(27.0)$ & $284(37.5)$ & \\
\hline Light activity & $245(6.8)$ & $200(7.0)$ & $45(6.0)$ & \\
\hline Moderate activity & $309(8.5)$ & $268(9.4)$ & $41(5.4)$ & \\
\hline Vigorous activity & $2009(55.5)$ & $1622(56.6)$ & $387(51.1)$ & \\
\hline Kidney problems & $3621[0]$ & $2864[0]$ & $757[0]$ & 0.093 \\
\hline Yes & $87(2.4)$ & $55(1.9)$ & $32(4.2)$ & \\
\hline No & $3534(97.6)$ & $2809(98.1)$ & $725(95.8)$ & \\
\hline Liver problems & $3621[0]$ & $2864[0]$ & $757[0]$ & 0.012 \\
\hline Yes & $84(2.3)$ & $53(1.9)$ & $31(4.1)$ & \\
\hline No & $3537(97.7)$ & $2811(98.1)$ & $726(95.9)$ & \\
\hline Total cholesterol (mg/dL) & $199.1 \pm 0.96$ & $198.0 \pm 1.03$ & $202.9 \pm 1.65$ & 0.0165 \\
\hline Direct HDL-cholesterol (mg/dL) & $53.9 \pm 0.32$ & $53.6 \pm 0.31$ & $54.8 \pm 0.61$ & 0.0535 \\
\hline LDL-cholesterol (mg/dL) & $115.9 \pm 1.63$ & $116.1 \pm 1.90$ & $115.1 \pm 2.31$ & 0.9266 \\
\hline Triglycerides (mg/dL) & $141.4 \pm 3.60$ & $133.9 \pm 3.27$ & $169.5 \pm 12.51$ & 0.0174 \\
\hline Serum C-reactive protein $(\mathrm{CRP})(\mathrm{mg} / \mathrm{dL})$ & $3621[0]$ & $2864[0]$ & $757[0]$ & $<.0001$ \\
\hline Undetectable (CRP<=0.01) & $300(8.3)$ & $261(9.1)$ & $39(5.2)$ & \\
\hline Elevated $(0.01<\mathrm{CRP}<=1)$ & $2941(81.2)$ & $2352(82.1)$ & $589(77.8)$ & \\
\hline Clinically raised $(\mathrm{CRP}>1)$ & $380(10.5)$ & $251(8.8)$ & $129(17.0)$ & \\
\hline Diabetes mellitus & $3621[0]$ & $2864[0]$ & $757[0]$ & $<.0001$ \\
\hline Yes & $334(9.2)$ & $191(6.7)$ & $143(18.9)$ & \\
\hline No & $3287(90.7)$ & $2673(93.3)$ & $614(81.1)$ & \\
\hline Hypertension & 3418 [203] & $2702[162]$ & $716[41]$ & $<.0001$ \\
\hline Normal & $1603(46.9)$ & $1339(49.6)$ & $264(36.9)$ & \\
\hline Pre-hypertension & $1465(42.9)$ & $1127(41.7)$ & $338(47.2)$ & \\
\hline Hypertension stage 1 & $283(8.3)$ & $194(7.2)$ & $89(12.4)$ & \\
\hline Hypertension stage 2 & $67(1.9)$ & $42(15)$ & $25(3.5)$ & \\
\hline Cardiovascular disease & $3621[0]$ & $2864[0]$ & $757[0]$ & $<.0001$ \\
\hline Yes & $262(7.2)$ & $125(4.4)$ & $137(18.1)$ & \\
\hline No & $3359(92.8)$ & $2739(95.6)$ & $620(81.9)$ & \\
\hline
\end{tabular}

*All values are weighted according to NHANES recommendations. Values correspond to mean \pm standard deviation or $\mathrm{n}(\%)$.

** Statistical significance level: 0.05

Light activity: over the last 30 days, walked or bicycled as part of getting to and from work, or school, or to do errands.

Moderate activity: over the last 30 days, activity for at least 10 minutes that causes only light sweating or a slight to moderate increase in breathing or heart rate.

Vigorous activity: over the last 30 days, activity that causes heavy sweating or large increases in breathing or heart rate.

Vigorous alcohol consumption: M: more than 14 drinks per week- F: more than 7 drinks per week.

Normal: systolic blood pressure $<120 \mathrm{~mm} \mathrm{Hg}$ and diastolic blood pressure $<80 \mathrm{~mm} \mathrm{Hg}$.

Pre-hypertension (at risk): $120 \mathrm{~mm} \mathrm{Hg} \leq$ systolic blood pressure $<140 \mathrm{~mm} \mathrm{Hg}$ or $80 \mathrm{~mm} \mathrm{Hg} \leq$ diastolic blood pressure $<90 \mathrm{~mm} \mathrm{Hg}$.

Hypertension stage 1: $140 \mathrm{~mm} \mathrm{Hg} \leq$ systolic blood pressure $<160 \mathrm{~mm} \mathrm{Hg}$ or $90 \mathrm{~mm} \mathrm{Hg} \leq$ diastolic blood pressure $<100 \mathrm{~mm} \mathrm{Hg}$.

Hypertension stage 2: systolic blood pressure $\geq 160 \mathrm{~mm} \mathrm{Hg}$ or diastolic blood pressure $\geq 100 \mathrm{~mm} \mathrm{Hg}$. 
Table 2. Associations of arthritis with cardiovascular disease by sex** (n=3621)

\begin{tabular}{|c|c|c|c|}
\hline & Odds Ratio & $95 \%$ CI & P value $* * *$ \\
\hline \multicolumn{4}{|l|}{ BIVARIATE ASSOCIATION } \\
\hline All participants $(\mathrm{n}=3621)$ & 4.81 & $3.66-6.31$ & $<.0001$ \\
\hline Males $(n=1856)$ & 4.5 & $3.12-6.41$ & $<.0001$ \\
\hline Females $(\mathrm{n}=1765)$ & 5.6 & $2.95-10.62$ & $<.0001$ \\
\hline \multicolumn{4}{|c|}{ MULTIVARIATE ASSOCIATION**** } \\
\hline \multicolumn{4}{|l|}{ Covariates } \\
\hline Age & 1.08 & $1.06-1.1$ & $<.0001$ \\
\hline \multicolumn{4}{|l|}{ Body Mass Index } \\
\hline Underweight $(\mathrm{BMI}<18.5)$ & 2.59 & $0.51-13.34$ & 0.2538 \\
\hline Normal $(18.5<=\mathrm{BMI}<24.9)$ & Reference & Reference & Reference \\
\hline Overweight $(24.9<=\mathrm{BMI}<30)$ & 1.7 & $1.17-2.44$ & 0.0052 \\
\hline Obese $(\mathrm{BMI}>=30)$ & 2.43 & $1.64-3.60$ & $<.0001$ \\
\hline \multicolumn{4}{|l|}{ Alcohol consumption } \\
\hline Light consumption & Reference & Reference & Reference \\
\hline Moderate consumption & 0.7 & $0.37-1.31$ & 0.2709 \\
\hline Vigorous consumption & 0.66 & $0.42-1.06$ & 0.0853 \\
\hline Total Cholestérol & 0.99 & $0.98-1.00$ & 0.0087 \\
\hline \multicolumn{4}{|l|}{ Arthritis } \\
\hline All participants $(\mathrm{n}=3621)$ & 2.01 & $1.38-2.94$ & 0.0003 \\
\hline Males (n1=1856) & 1.94 & $1.32-2.87$ & 0.0007 \\
\hline Females (n2=1765) & 2.38 & $0.96-5.86$ & 0.0598 \\
\hline
\end{tabular}

*All values are weighted according to NHANES recommendations.

$* *$ p value of the interaction between arthritis and sex is 0.729 , not statistically significant $* * *$ The level of significance is set below 0.05

$* * * *$ Multivariate model adjusted for age, sex, total cholesterol level, body mass index and alcohol consumption

OR (95\% CI): Odds Ratios with 95\% confidence intervals

Light alcohol consumption: $\mathrm{M}:<=3$ drinks per week- $\mathrm{F}:<=3$ drinks per week

Moderate alcohol consumption: M: between 3 and 14 drinks per week- F: between 3 and

7 drinks per week

Vigorous alcohol consumption: M: more than 14 drinks per week- F: more than 7 drinks per week

without arthritis. The confidence interval (0.96 - 5.86) included the null value, but the $\mathrm{p}$ value was at the limit of statistical significance (0.0598). The confidence intervals for men and women overlapped, and the p-value of the interaction term between arthritis and sex was not statistically significant (0.729).

\section{Discussion}

In this large cross-sectional study, we observed a two-fold statistically significant increased association between self-reported diagnoses of arthritis and CVD in a cohort of non-institutionalized U.S. adults. This association was not modified by sex.

Our results are consistent with those of previous studies on the association between arthritis and CVD [12,13,27,28]. For instance, Soubrier et al. have reported an adjusted OR of 1.7 (95\% CI: 1.5-6.9) [13]. Inflammation has been long recognized as a common component of both inflammatory arthritis and CVD, especially those related to atherosclerosis $[12,35]$. According to a meta-analysis of 15 studies that included 358,944 people, the risk of CVD was significantly increased by $24 \%$ in patients with osteoarthritis (OA) compared with the general population [36]. In a longitudinal study of 4,265 persons without CVD followed over 8 years, participants with OA at baseline had a significantly increased risk of CVD during follow-up of about 27\% [37]. The authors mentioned that shared conditions between OA and CVD such as hypertension, obesity, high cholesterol and diabetes, along with inactivity and the use of nonsteroidal anti-inflammatory drugs (NSAIDs), can explain the increased risk [36,37]. Schieir et al. [38] reported consistent increased risk of MI across multiple common arthritis disease types. In studies adjusted for age and sex, risk of incident MI was significantly increased in participants with rheumatoid arthritis, gout, psoriatic arthritis, and OA; there was also a trend towards increased risk in ankylosing spondylitis. Like us, Meek et al. also found hypertension and obesity to be overrepresented among patients with chronic rheumatic diseases. However, they further reported an association of cigarette smoking with arthritis that we have not seen [4].

As in other studies $[4,12,13,27]$, we found that age, total cholesterol concentrations, obesity and alcohol consumption were actual confounding factors on the studied association. For example, untreated rheumatoid arthritis patients have dyslipidemia, and total cholesterol concentrations usually decreases with decreasing disease activity [4]. In addition, decreased cholesterol concentrations lead to a decrease in the atherogenic index, lowering the atherosclerotic risk development [13]. As for obesity, it is a well-known risk factor for CVD [28], and overweight is almost uniformly present among patients with rheumatic cardiovascular diseases [4].

In contrast, while hypertension is a classical CVD risk factor that is highly prevalent in people with arthritis $[4,12,13,27,28]$ it was not retained in our analyses as a confounding factor. It might be that our hypertensive participants were under hypertensive treatments and thus, hypertension did not influence the association.

This study has several important strengths. It is based on data collected from a large representative sample of the U.S. adult population, providing high statistical power. It explored a new topic and advances knowledge about the association between inflammatory arthritis and CVD. In addition, some variables (such as smoking status and cholesterol levels) were measured physiologically and clinically at the same time, which provided data that are more accurate.

Since we used a cross-sectional design, our first study limitation lies in the fact we could not establish the directionality of the associations. Thus, we cannot know whether the relationship between arthritis and CVD is causal. Also, data were derived from a survey conducted in 2005-2006 and results may be different due to advancements in arthritis treatment.

Another important limitation is linked to some variable's measurement. Several variables were self-reported by questionnaire, holding some potential for information biases, most likely nondifferential; this probably resulted in an underestimation of the strength of the association between arthritis and CVD. Another non-differential information bias is related to the measure of the independent variable. Our interest concerned the inflammatory arthritis diseases only. However, NHANES data provided a global measure for the arthritis disease; the term "arthritis" was not specific and included osteoarthritis, rheumatoid arthritis, spondylarthritis, psoriatic arthritis, etc. We were forced to consider arthritic diseases globally without being able to separate inflammatory arthritis from non-inflammatory ones.

We have controlled for several confounders identified in the literature and we believe to have adjusted for the most important ones. However, residual confounding linked to factors such as nutrition and drug used is nevertheless still a possibility. Also, although CRP is mentioned in the literature as a risk factor for CVD [13,27,28], we thought it is likely to be on the pathway between arthritis and CVD, and thus did not adjust for it. To validate this approach, however, we introduced it in our final regression model: it did not bring about a change of $>10 \%$ in the ORs and would thus not have been retained as a confounder anyway. 
As for external validity, participants were from the U.S. only. Given important differences in health care systems between the U.S. and many other countries, this potentially limits the generalizability of our results.

It is important to mention that the OR is not a valid estimate of the relative risk when the prevalence of the disease under scrutiny is high like it is in this study.

We found a difference in ORs between men and women that could have signed a stronger association in women. However, this difference was small in magnitude, confidence intervals of the ORs in men and women overlapped, and the interaction term between arthritis and sex was not statistically significant; thus, we must conclude that sex does not modify the association between arthritis and CVD.

Although the association between arthritis and CVD is well described in the literature, in both Europe and the U.S. there is a deficiency in CVD risk management among individuals with arthritis. Compared to the general population, patients with rheumatoid arthritis are less likely to benefit from CVD preventive measures [28]. Healthcare providers underuse the systematic evaluation tools of CVD risk among patients with rheumatoid arthritis. In fact, the interdisciplinary team managing CVD risk in patients with rheumatoid arthritis usually includes a cardiologist, a rheumatologist and a primary care provider, and confusion does often exist among them about who should evaluate CVD risk factors and provide related lifestyle recommendations and medical interventions [28]. It is important to translate our findings into the daily clinical practices of those who treat patients with inflammatory arthritis to help them provide better patient primary CVD prevention treatment. In addition, healthcare professionals should be more aggressive in addressing CVD risk factors among women who have often atypical presentation of CVD. This is because mainly small vessels are involved in the disease rather than major ones $[39,40]$, leading to different risk profile when diagnosed with CVD [19].

Future research based on longitudinal studies is fundamental to confirm our results. In addition, because the concentrations of several biomarkers, especially vitamins and minerals, differ between men and women, affecting thus the manifestation of both diseases, it would be important to study the effect modification of sex combined to those biomarkers, on the association between arthritis and CVD.

\section{Conclusions}

Using data from a large survey conducted in the U.S., we observed a significant association between arthritis and CVD that was not modified by sex. These findings have important clinical implications for the management of patients with arthritis diseases. However, as our study was cross-sectional, further longitudinal studies are required to validate our conclusion.

\section{Authors' contributions}

RS participated in conception and design, acquisition of data, analysis, interpretation, and drafting the manuscript. CED participated in conception and design, interpretation, and critical revision of the manuscript. DL participated in interpretation and critical revision of the manuscript. All authors read and approved the final manuscript.

\section{Conflicts of interest}

The authors declare that they have no competing interests.

\section{Ethics approval}

The ethics committee of the Laval University (CERUL: https:// www.cerul.ulaval.ca) validated that this type of research does not need ethical approval since it is based exclusively on secondary use of anonymous data, and information used is legally available to the public and adequately protected by the law.

\section{Consent to participate}

All participants provided written informed consent before participating to NHANES 2005-2006.

\section{Availability of data and materials}

The datasets supporting the conclusions of this article are available in the NHANES 2005-2006 website (http://wwwn.cdc.gov/nchs/ nhanes/search/nhanes05_06.aspx

\section{References}

1. Mendis S, Puska P, Norrving B, World Health Organization, World Heart Federation, et al. (2011) Global atlas on cardiovascular disease prevention and control.

2. van Holten TC, Waanders LF, de Groot PG, Vissers J, Pasterkamp G, et al. (2013) Circulating Biomarkers for Predicting Cardiovascular Disease Risk; a Systematic Review and Comprehensive Overview of Meta-Analyses. PLoS ONE 8: 1-8. [Crossref]

3. WHO (2014) Cardiovascular diseases.

4. Meek IL, Picavet HS, Vonkeman HE, Verschuren WM, van de Laar MA (2013) Increased cardiovascular risk factors in different rheumatic diseases compared with the general population. Rheumatology 52: 210-216. [Crossref]

5. Ren Y, Zeng RX, Li JJ, Guo LH, He Y, et al. (2015) Relation of C-reactive protein and new-onset atrial fibrillation in patients with acute myocardial infarction: A systematic review and meta-analysis. Int J Cardiol 190: 268-270. [Crossref]

6. Yoshizaki T, Umetani K, Ino Y, Takahashi S, Nakamura M, et al. (2012) Activated Inflammation is Related to the Incidence of Atrial Fibrillation in Patients with Acute Myocardial Infarction. Intern Med 51: 1467-1471. [Crossref]

7. Davenport G (2004) Rheumatology and musculoskeletal medicine. British Journal of General Practice 54: 457-464.

8. van der Meer I, De Maat M, Kiliaan A, van der Kuip D, Hofman A, et al. (2003) The Value of C-Reactive Protein in Cardiovascular Risk Prediction: The Rotterdam Study. Arch Intern Med 163: 1323-1328. [Crossref]

9. Arthritis Society (2019) About arthritis.

10. Centers for Disease Control and Prevention (2019) Arthritis.

11. Mease P, Garg A, Gladman D, Helliwell P (2013) Development of Simple Clinical Criteria for the Definition of Inflammatory Arthritis, Enthesitis, Dactylitis, and Spondylitis: A Report from the GRAPPA 2012 Annual Meeting. J Rheumatol 40: 1442-1445. [Crossref]

12. Hollan I, Meroni PL, Ahearn JM, Cohen Tervaert JW, Curran S, et al. (2013) Cardiovascular disease in autoimmune rheumatic diseases. Autoimmun Rev 12: 10041015. [Crossref]

13. Soubrier M, Chamoux NB, Tatar Z, Couderc M, Dubost JJ, et al. (2014) Cardiovascular risk in rheumatoid arthritis. Joint Bone Spine 81: 298-302.

14. Li JJ, Fang CH (2004) C-reactive protein is not only an inflammatory marker but also a direct cause of cardiovascular diseases. Med Hypotheses 62: 499-506. [Crossref]

15. Rhodes B, Furnrohr BG, Vyse TJ (2011) C-reactive protein in rheumatology: biology and genetics. Nat Rev Rheumatol 7: 282-289. [Crossref]

16. Regitz-Zagrosek V, Lehmkuhl E, Weickert M (2006) Gender differences in the metabolic syndrome and their role for cardiovascular disease. Clin Res Cardiol 95: 136-147. [Crossref]

17. Mendis S, Puska P, Norrving B (2011) Global Atlas on Cardiovascular Disease Prevention and Control. World Health Organization. Geneva.

18. Rosen SE, Henry S, Bond R, Pearte C, Mieres JH (2015) Sex-Specific Disparities in Risk Factors for Coronary Heart Disease. Curr Atheroscler Rep 17: 523. [Crossref] 
19. Lawton JS (2011) Sex and gender differences in coronary artery disease. Semin Thorac Cardiovasc Surg 23: 126-130. [Crossref]

20. Sokka T, Toloza S, Cutolo M, Kautiainen H, Makinen H, et al. (2009) Women, men, and rheumatoid arthritis: analyses of disease activity, disease characteristics, and treatments in the QUEST-RA study. Arthritis Res Ther 11: R7. [Crossref]

21. Ahlmen M, Svensson B, Albertsson K, Forslind K, Hafstrom I, et al. (2010) Influence of gender on assessments of disease activity and function in early rheumatoid arthritis in relation to radiographic joint damage. Ann Rheum Dis 69: 230-233. [Crossref]

22. Centers for Disease Control and Prevention (2019) National Center for Chronic Disease Prevention and Health Promotion, Division of Population Health.

23. Möller-Leimkühler AM (2007) Gender differences in cardiovascular disease and comorbid depression. Dialogues Clin Neurosci 9: 71-83. [Crossref]

24. The EUGenMed, Cardiovascular Clinical Study Group, Regitz-Zagrosek V, OerteltPrigione S, Prescott E, et al. (2016) Gender in Cardiovascular Diseases: Impact on Clinical Manifestations, Management, and Outcomes. Eur Heart J 37: 24-34.

25. Centers for Disease Control and Prevention (2014) National Health and Examination Survey Overview. Hyattsville, MD: US Department of Health and Human Services, Centers for Disease Control and Prevention.

26. Kendrick J, Targher G, Smits G, Chonchol M (2009) 25-Hydroxyvitamin D deficiency is independently associated with cardiovascular disease in the Third National Health and Nutrition Examination Survey. Atherosclerosis 205: 255-260. [Crossref]

27. John H, Kitas G (2012) Inflammatory arthritis as a novel risk factor for cardiovascular disease. Eur J Intern Med 23: 575-579. [Crossref]

28. Semb AG, Rollefstad S, van Riel P, Kitas GD, Matteson EL, et al. (2014) Cardiovascular disease assessment in rheumatoid arthritis: a guide to translating knowledge of cardiovascular risk into clinical practice. Annals of the rheumatic diseases 73: 1284-1288.

29. van Breukelen-van der Stoep DF, Klop B, van Zeben D, Hazes JM, Castro Cabezas M (2013) Cardiovascular risk in rheumatoid arthritis: how to lower the risk? Atherosclerosis 231: 163-172. [Crossref]
30. Jacob P, Yu L, Wilson M, Benowitz N (1991) Selected ion monitoring method for determination of nicotine, cotinine and deuterium-labeled analogs: absence of an isotope effect in the clearance of (S)-nicotine-3',3'-d2 in humans. Biol Mass Spectrom 20: 247-252. [Crossref]

31. Jarvis MJ, Russell MA, Benowitz NL, Feyerabend C (1988) Elimination of cotinine from body fluids: implications for noninvasive measurement of tobacco smoke exposure. Am J Public Health 78: 696-698. [Crossref]

32. Kwiterovich PO (2008) Triglycrides in Serum using the Hitachi 717 NHANES 2005-2006.

33. Prevention CfDCa (2010) Data Documentation, Codebook, and Frequencies-Cholesterol Total (TCHOL_D) National Health and Nutrition Examination Survey 2005-2006.

34. Mirel L, Mohadjer L, Dohrmann SM, Clark J, Burt VL, et al. (2013) National Health and Nutrition Examination Survey: Estimation procedures, 2007-2010.Vital Health Stat 2: 1-17. [Crossref]

35. Goshayeshi L, Saber H, Sahebari M, Rezaieyazdi Z, Rafatpanah H, et al. (2012) Association between metabolic syndrome, BMI, and serum vitamin D concentrations in rheumatoidarthritis. Clin Rheumatol 31: 1197-1203. [Crossref]

36. Wang H, Bai J, He B, Hu X, Liu D (2016) Osteoarthritis and the risk of cardiovascular disease: a meta-analysis of observational studies. Sci Rep 6: 39672. [Crossref]

37. Veronese N, Stubbs B, Solmi M, Smith TO, Reginster JY, et al. (2018) Osteoarthristis Increases the Risk of Cardiovascular Disease: Data from the Osteoarthritis Initiative. $J$ Nutr Health Aging 22: 371-376. [Crossref]

38. Schieir O, Tosevski C, Glazier RH, Hogg-Johnson S, Badley EM (2017) Incident myocardial infarction associated with major types of arthritis in the general population: a systematic review and meta-analysis. Ann Rheum Dis 76: 1396-1404. [Crossref]

39. Szerlip M, Grines CL (2009) Sex differences in response to treatments for chronic coronary artery disease. Rev Cardiovasc Med 10: 14-23. [Crossref]

40. Kent JA, Patel V, Varela NA (2012) Gender disparities in health care. Mt Sinai J Med 79: 555-559. [Crossref]

Copyright: (C2021 Saade R. This is an open-access article distributed under the terms of the Creative Commons Attribution License, which permits unrestricted use, distribution, and reproduction in any medium, provided the original author and source are credited. 